\title{
Optically-guided scalpel with light-scattering module for carpal tunnel surgical procedure via minimally invasive surgery
}

\author{
Ming-Che Chiang ${ }^{\mathrm{a}}$ and Ching-Cheng Huang, , $^{\text {* }}$ \\ ${ }^{a}$ Department of Neurosurgical Taichung Veterans General Hospital, Taiwan \\ ${ }^{b}$ Regional R\&D Service Department, Metal Industries Research \& Development Centre, 6F, No. 162- \\ 24, Sec. 3, Hsin-Yi Rd., Taipei 10658, Taiwan \\ ${ }^{c}$ Department of Biomedical Engineering, Ming-Chuan University, 5 De Ming Rd., Gui Shan District, \\ Taoyuan, 333, Taiwan
}

\begin{abstract}
A novel technique and product for carpal tunnel microscopic surgical procedures using a modified medical device containing a head as a surgical scalpel under light guidance were designed and studied. The novel design of the medical device was developed and applied in a new carpal tunnel microscopic surgical procedure in place of the traditional carpal tunnel surgical procedure. Biological and clinical evaluations of carpal tunnel surgical procedure using the designed optically guided medical device were studied. For commercialized reasons, some guidance was considered, such as ISO 109931:2009(E), for the biological evaluation of the device. Furthermore, a clinical evaluation was carried out. The designed optically guided medical device could provide a powerful medical device for carpal tunnel syndrome and related applications.
\end{abstract}

Keywords: Biological evaluation, clinical evaluation, carpal tunnel, microscopic surgical procedure, scalpel

\section{Introduction}

A novel optically guided medical device with a scalpel and light-scattering module was designed for clinical needs of carpal tunnel surgical procedures via minimally invasive surgery (MIS). The wrist is surrounded by a band of fibrous tissue that supports the joint. The tight space between the wrist bone and the fibrous band is called the carpal tunnel. The median nerve passes through the carpal tunnel to receive sensations from the thumb, index, and middle fingers. Hence, any condition that causes swelling or a change in the position of the tissue within the carpal tunnel can repress and damage the median nerve. Repression and irritation of the median nerve can cause numbness and tingling of the thumb, index, and middle fingers, which is a clinical condition known as carpal tunnel syndrome. In this study, a novel technique and medical device for carpal tunnel microscopic surgical procedures through a modified medical device containing a head as a scalpel using light guidance was proposed.

\footnotetext{
*Address for correspondence: Ching-Cheng Huang, Regional R\&D Service Department, Metal Industries Research \& Development Centre, 6F, No. 162-24, Sec. 3, Hsin-Yi Rd., Taipei 10658, Taiwan. Tel.: +886-988-915063; Fax: +886-3-3593840; E-mail: junas.tw@yahoo.com.tw.
} 
The novel design was developed and applied in carpal tunnel microscopic surgical procedures in place of traditional carpal tunnel surgical procedures. For the design of new medical applications, the selection of materials should be carefully considered. Several reports for the design, synthesis, and preparation of new functional materials were studied to find suitable materials for biomedical applications such as polyacrylate, polyester, polyamide, polyimide, polyester, polynorborene, polytetrafluoroethylene, polydiphenylacetylenes, and polymeric resins [1-16]. Also, surface modification technology was considered to change the surface microenvironment of the materials [17, 18]. Finally, biological and clinical evaluations of the materials were considered for the application and design.

\section{Materials and methods}

\subsection{New design and fabrication of optically guided medical device with scalpel}

A new design and fabrication of an optically guided medical device with a scalpel were studied. The device contained a scalpel with a blade, an optically guided system with scattering propagation, a power controller, and a connectable power supply.

\subsection{Biological evaluation of medical devices}

For commercialized reasons, some guidance must be considered, such as ISO 10993-1:2009 (E), for biological evaluation of medical devices part 1: evaluation and testing with a risk management process, ISO 10993-5:2009 for biological evaluation of medical devices part 5: test for in vitro cytotoxicity and so on.

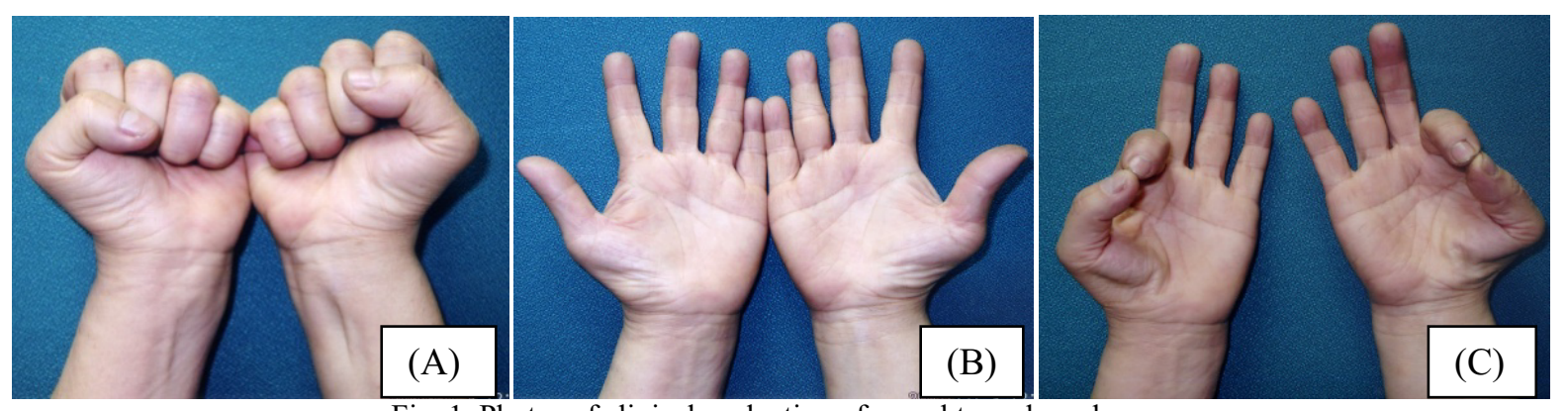

Fig. 1. Photos of clinical evaluation of carpal tunnel syndrome.

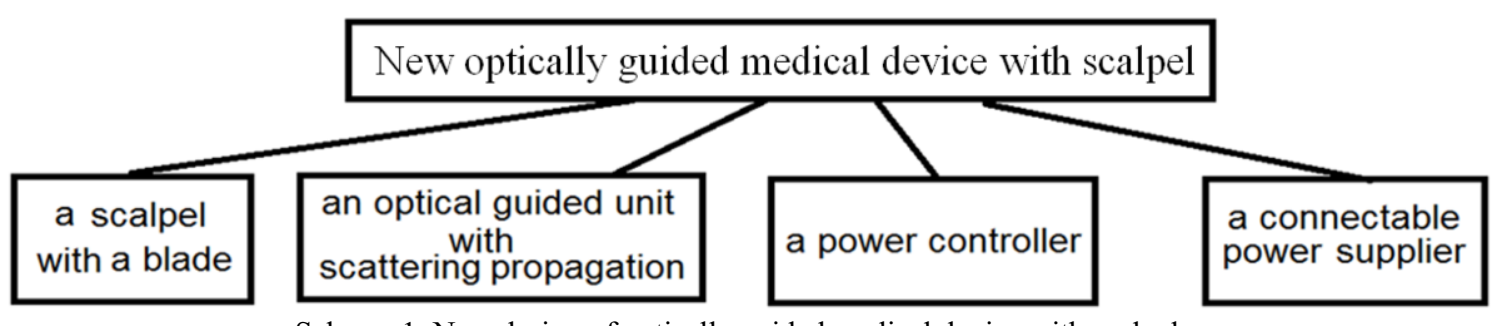

Scheme 1. New design of optically guided medical device with scalpel. 

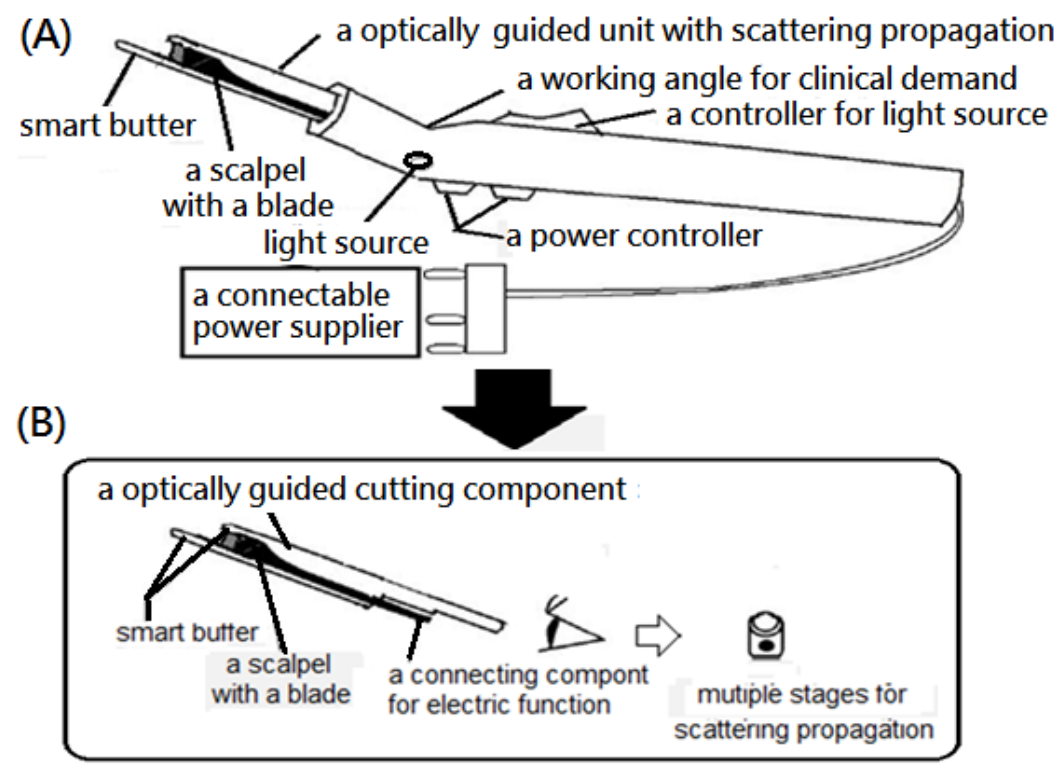

Fig. 2. (A) New design of optically guided medical device with scalpel and its key functional components; (B) New design of optically guided cutting component and multiple stages for scattering propagation.

(A)
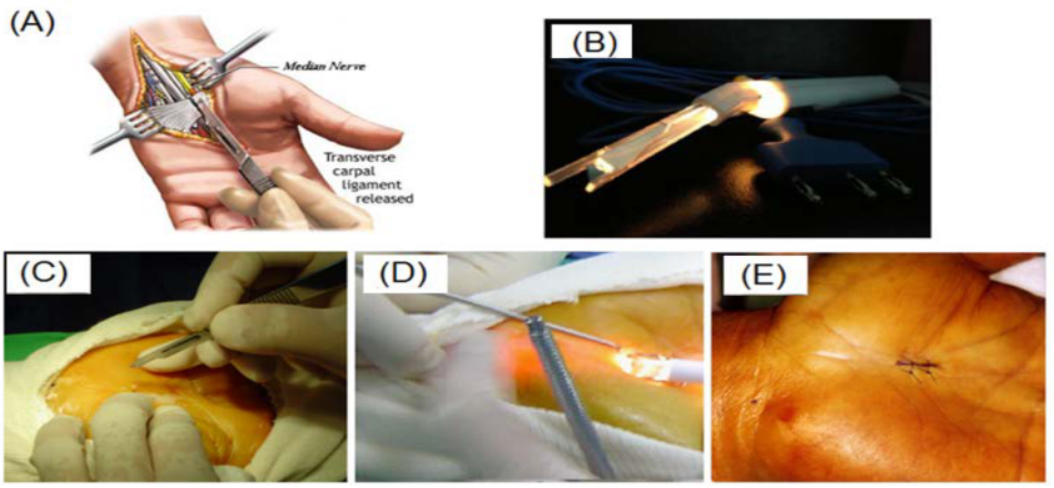

Fig. 3. (A) Traditional design of medical devices for carpal tunnel surgical procedure [http://www.medicinenet.com/carpal_tunnel_syndrome/article.htm]; (B) New design of optically guided medical device with scalpel in this study; (C)-(E) New design of optically guided medical device with scalpel employed in carpal tunnel surgical procedure.

\section{Results and discussion}

\subsection{New design of fabrication of optically guided medical device with scalpel for carpal tunnel syndrome}

In this study, traditional and conventional medical methods for carpal tunnel surgical procedures were modified, and a novel optically guided medical device with a scalpel was designed for the surgical repair of carpal tunnel syndrome (Figure 1 and Scheme 1). Photos of a clinical evaluation of carpal tunnel syndrome are shown in Figure 1. Numbness and tingling of the thumb, index, and middle fingers are clinical evaluations for carpal tunnel syndrome. To meet clinical demand, a new design and 
fabrication of an optically guided medical device with a scalpel was necessary. Some essential components were considered for the clinical need for carpal tunnel surgical procedure (Scheme 1). Therefore, a new medical device was designed, which had optically guided components with a scalpel, an optically guided system with scattering propagation, a power controller, and a connectable power supply (Figure 2). The scattering propagation was achieved by using the design of multiple stages in an optically guided cutting unit (Figure 2). The electrical supply, which provided optical guidance and electric cutting, was also part of the medical device, as shown in Scheme 1. The relatively large operating area observed by traditional carpal tunnel surgical procedures is shown in Figure 3(A). The fabrication of an optically guided medical device with a scalpel for the surgical repair of carpal tunnel syndrome was carried out, and the new medical device is shown in Figures 3(B)-3(E).

\subsection{Characterization of the designed optically guided medical device with scalpel}

A modified medical device containing a head as a surgical scalpel under optical guidance was designed. The newly designed head as a surgical scalpel is shown in Figure 4. Multiple stages for light scattering propagations were designed. The light scattering propagation occurred because of the light transfer delay among different light waves, which satisfied the clinical demand during carpal tunnel surgical procedure to show the light passing under the skin, as shown in Figure 3(D).

Polyacrylate was designed as the material for optical guidance in place of glass. Thermal deformation temperature was obtained at $93.9^{\circ} \mathrm{C}$ under $0.455 \mathrm{MPa}$ (ASTM D648-07B). Furthermore, an experiment involving skin and flesh of a pig was performed under optical guidance using the created and modified medical device. Pig skin and flesh were successfully cut using the designed modified medical device, as shown in Figure 5.
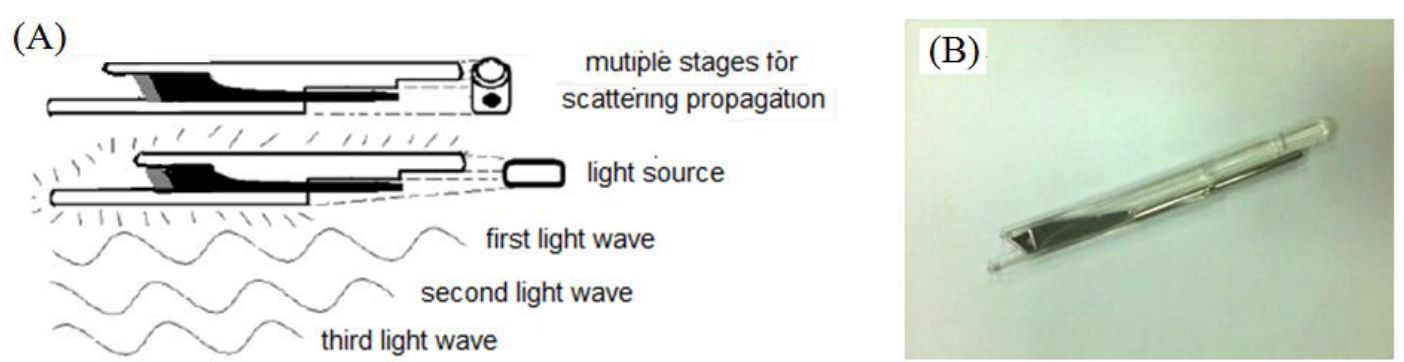

Fig. 4. (A) The schematic diagram; (B) Photo of new designed head as a surgical scalpel in the modified medical device and multiple stages for light scattering propagation.

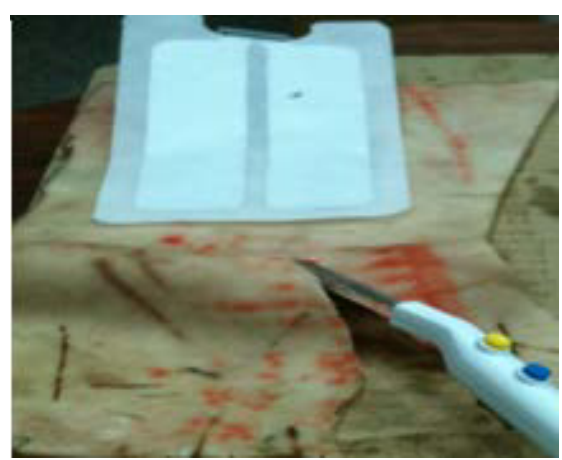

Fig. 5. Pig skin cutting test using the medical device containing a head as a surgical scalpel was performed under optical guidance. 
Table 1

The average number of the cell layers

\begin{tabular}{ll}
\hline & Average number of cell layer \\
\hline Negative control groups & $3.66 \pm 0.21$ \\
& $1.10 \pm 0.13$ \\
Control groups & $3.71 \pm 0.05$ \\
Sample & \\
\hline
\end{tabular}

Table 2

Design of clinical evaluation table of carpal tunnel surgical procedure using the new optically guided medical device with a scalpel and light-scattering modules

\begin{tabular}{|l|l|}
\hline B) & Time after carpal tunnel surgical procedure \\
\hline Suggesting Procedure:
\end{tabular}

\subsection{Biological evaluation of medical devices}

For commercialized reasons, some guidance must be considered, such as ISO 10993-1:2009(E) for the biological evaluation of medical devices. Some results for the biological evaluation of the designed optically guided medical device with a scalpel and light-scattering modules were employed in this study (Table 1). The average number of cell layers was obtained and excellent biocompatible properties were shown for the new medical equipment (Table 1). For specific clinic needs, the electronic power could be applied to the scalpel module of the medical device.

\subsection{Clinical evaluation of carpal tunnel surgical procedure with medical device}

The clinical evaluation of carpal tunnel surgical procedures with the new light guided medical device was studied by a design of clinical evaluation table of carpal tunnel surgical procedure. The red part shown in Table 2(A) involves the muscular dystrophy position of carpal tunnel syndrome. The red marks of sutures, as shown in Table 2(B), indicate the incision site of the new carpal tunnel surgical procedure. Also, the "Suggesting Procedure before carpal tunnel surgical procedure" and "Time after carpal tunnel surgical procedure" could be reported in the designed table. 


\section{Summary}

This study provided the novel design and fabrication of an optically guided medical device with a light-scattering module for carpal tunnel syndrome repair. Furthermore, the study provided a comparison of traditional carpal tunnel surgical procedure using surgical scalpels against the carpal tunnel microscopic surgical procedure using the novel light guided medical device. Biological and clinical evaluations were carried out, and the designed light-guided medical device proved to be a powerful medical device for carpal tunnel syndrome and related applications.

\section{Acknowledgments}

The authors gratefully acknowledge Shi-Heng Enterprise Co. Ltd., Taiwan and the Ministry of Economic Affairs, Taiwan for their financial supports. Also, the authors acknowledge for Mr. Dean Lee for his technical assistance.

\section{References}

[1] T.T. Reddy, A. Kano, A. Maruyama, M. Hadano and A. Takahara, Thermosensitive transparent semi-interpenetrating polymer networks for wound dressing and cell adhesion control, Biomacromolecules 9 (2008), 1313-1321.

[2] D.J. Liaw, C.C. Huang and B.Y. Liaw, Synthesis and properties of polyurethanes based on bisphenol-s derivatives, Polymer 39 (1998), 3529-3535.

[3] D.J. Liaw, C.C. Huang, H.C. Sang and E.T. Kang, Photophysical and solution properties of naphthalene-labeled styrene / N, N-dimethyl maleimido propylammonium propane sulfonate copolymer, Langmuir 15 (1999), 5204-5211.

[4] D.J. Liaw, C.C. Huang, H.C. Sang and P.L. Wu, Macromolecular microstructure, reactivity ratio and viscometric studies of water-soluble cationic and / or zwitterionic copolymers, Polymer 41 (2000), 6123-6131.

[5] M.T. Cerqueira, R.P. Pirraco, T.C. Santos, D.B. Rodrigues, A.M. Frias, A.R. Martins, R.L. Reis and A.P. Marques, Human adipose stem cells cell sheet constructs impact epidermal morphogenesis in full-thickness excisional wounds, Biomacromolecules 14 (2013), 3997-4008.

[6] D.J. Liaw, C.C. Huang and P.L. Wu, Synthesis and characterization of novel diblock copolymers of 5-(N-carbazoyl methyl)bicyclo[2.2.1] hept-2-ene and 5-(Phthalimide methyl)bicyclo[2.2.1] hept-2-ene via living ring-opening metathesis polymerization, Polymer 42 (2001), 9371-9377.

[7] D.J. Liaw, W.H. Chen and C.C. Huang, Synthesis and characterization of new organosoluble poly (ether-imide)s derived from various novel bis(ether anhydride)s, Polyimides and Other High Temperature Polymers 2 (2003), 47-70.

[8] A. Makino, K. Kurosaki, M. Ohmae and S. Kobayashi, Chitinase-catalyzed synthesis of alternatingly N-deacetylated chitin: A chitin-chitosan hybrid polysaccharide, Biomacromolecules 7 (2006), 950-957.

[9] C. Karakasyan, M. Legros, S. Lack, F. Brunel, P. Maingault, G. Ducouret and D. Hourdet, cold gelation of alginates induced by monovalent cations, Biomacromolecules 11 (2010), 2966-2975.

[10] S.V. Vlierberghe, V. Cnudde, P. Dubruel, B. Masschaele, A. Cosijns, I.D.P. Patric, J.S. Jacobs, L.V. Hoorebeke, J.P. Remon and E. Schacht, Porous gelatin hydrogels: 1. cryogenic formation and structure analysis, Biomacromolecules 8 (2007), 331-337.

[11]D.J. Liaw, T.P. Chen and C.C. Huang, Macromolecules 38 (2005), 3533-3538.

[12] M. Kumar, K.J. Sanford, W.A. Cuevas, M.D. Katharine, D. Collier and N. Chow, Designer protein-based performance materials, Biomacromolecules 7 (2006), 2543-2551.

[13] T. Katsumata, M. Maitani, C.C. Huang, M. Shiotsuki and T. Masuda, Synthesis and properties of various poly (diphenylacetylenes) containing tert-amine moieties, Polymer 49 (2008), 2808-2816.

[14] M. Hrynyk, M. Martins-Green, A.E. Barron and R.J. Neufeld, Alginate-PEG sponge architecture and role in the design of insulin release dressings, Biomacromolecules 13 (2012), 1478-1485.

[15] D.J. Liaw, C.C. Huang and E.T. Kang, Effect of architecture and environments on polymeric molecular assemblies of novel amphiphilic diblock copolynorbornenes with narrow polydispersity via living ring-opening metathesis polymerization (ROMP), Journal of Polymer Science Part A: Polymer Chemistry 44 (2006), 2901-2911. 
[16]D.J. Liaw, C.C. Huang and J.Y. Ju, Novel star-like multifunctional polymeric materials with predominant cis microstructures derived from $\alpha$-norbornenyl macromonomer and stable macroinitiator via ring-opening metathesis polymerization and atom transfer radical polymerization, Journal of Polymer Science Part A: Polymer Chemistry 44 (2006), 3382-3392.

[17] K.L. Neoh, C.C. Tan, C.C. Huang and D.J. Liaw, Surface structures and adhesive-free adhesion characteristics of polyaniline films after modification by graft copolymerization, Macromolecules 30 (1997), 3354-3362.

[18] G. Zhai, S.C. Toh, W.L. Tan, E.T. Kang, K.G. Neoh, C.C. Huang and D.J. Liaw, Poly (vinylidene fluoride) with grafted zwitterionic polymer side chains for electrolyte-responsive microfiltration membranes, Langmuir 19 (2003), 7030-7037. 